\title{
TROMBO EN LA AORTA APARENTEMENTE SANA, EL PODER DE LA ANTICOAGULACIÓN
}

\author{
THROMBUS IN APPARENTLY HEALTHY AORTA; THE POWER \\ OF ANTICOAGULATION
}

YU SHAN LIN', DIANA GUTIÉRREZ', EZEQUIEL LERECH', LUCIANO OCAMPOS², JULIETA SORICETTI³, DANIEL ORQUERA

\section{RESUMEN}

Los trombos móviles en la aorta sana en ausencia de enfermedad constituyen una entidad poco frecuente, cuya sospecha clínica suele aparecer luego de eventos embólicos. La ecocardiografía cardíaca transesofágica constituye una herramienta de gran utilidad para el diagnóstico. La forma más frecuente de presentación es la embolización arterial a predominio del miembro superior izquierdo. A continuación, se presenta un caso de una paciente con trombo móvil en la aorta ascendente sintomática por impotencia funcional y frialdad en el miembro superior izquierdo al esfuerzo y ausencia del pulso, la cual requirió anticoagulación con posterior resolución.

Palabras clave: trombosis, aórtica torácica, anticoagulantes.

\section{ABSTRACT}

Mobile thrombi in the healthy aorta in the absence of disease constitute a rare entity, whose clinical suspicion usually appears after embolic events. Transesophageal cardiac echocardiography is a very useful tool for diagnosis. The most frequent form of presentation is arterial embolization with predominance of the left upper limb. Wepresent a case of a patient with a mobile thrombus in the as cending symptomatic aorta due to functional impotence and coldness in the left upper limb due to stress and absence of the pulse that required anticoagulation, and was subsequently solved.

Keywords: thrombosis, thoracic aorta, anticoagulant.

REVISTA CONAREC 2018;33(145):194-196| DOI:10.32407/RCON/2018145/0194-0196

\section{INTRODUCCIÓN}

El diagnóstico de trombos móviles en la aorta sana, ausente de enfermedad ateroesclerótica y aneurismática, es poco frecuente, de etiología compleja que usualmente suele sospecharse luego de eventos embólicos con una estrategia de tratamiento aún desconocida ${ }^{1,2}$ Los métodos no invasivos de imágenes permiten identificar el foco

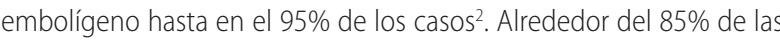
embolias arteriales tienen origen cardíaco, siendo la ecocardiografía transesofágica (ETE) de gran utilidad porque permite un examen exhaustivo tanto del corazón como de la aorta torácica' (Figura 1). La mayoría de los trombos proceden de la aorta abdominal y asientan en aneurismas o lesiones arterioescleróticas, aunque también se ha encontrado en zonas aparentemente sanas ${ }^{3,4}$.

La presentación clínica más frecuente es la embolígena, siendo la más habitual en las extremidades con cierto predominio por el miembro superior izquierdo $(\mathrm{MSI})^{5}$. Sin embargo, puede afectar cualquier parte del organismo.

1. Residente de Cardiología.

2. Jefe de residentes de Cardiología

3. Médico de planta de Cardiología.

Servicio de Cardiología, Hospital General de Agudos Carlos G. Durand. Ciudad Autónoma de Buenos Aires, Rep. Argentina.

$\triangle$ Correspondencia:Dr.Lin Yu Shan.Río de Janeiro 595, CABA. linyushan25@gmail.com

Los autores declaran no poseer conflictos de intereses.

Recibido: 01/11/2017 | Aceptado: 05/06/2018

\section{CASO CLÍNICO}

Paciente de sexo femenino, de 47 años, sin factores de riesgo cardiovasculares y sin antecedentes clínicos, oriunda de la zona rura de Santiago del Estero, consultó ambulatoriamente al Servicio de Cardiología para la realización de riesgo quirúrgico para una histerectomía por hipermenorrea secundaria a miomas. Al interrogatorio refirió presentar desde hace 3 meses parestesias y paresia moderada asociada a impotencia funcional y frialdad en MSI al esfuerzo. En

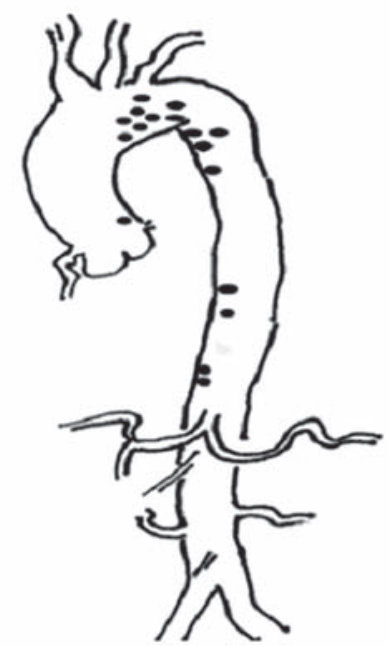

Figura 1. Sitios de localización más frecuentes de trombos en la aorta. Modificado de Pagn S, Trivedi J, Ganzel BL, Williams M, Kapoor N, Ross C et al. Thoracic aortic mobile thrombus: is there a role for early surgical intervention?. Ann Thorac Surg 2011;91(6):1875-81. 


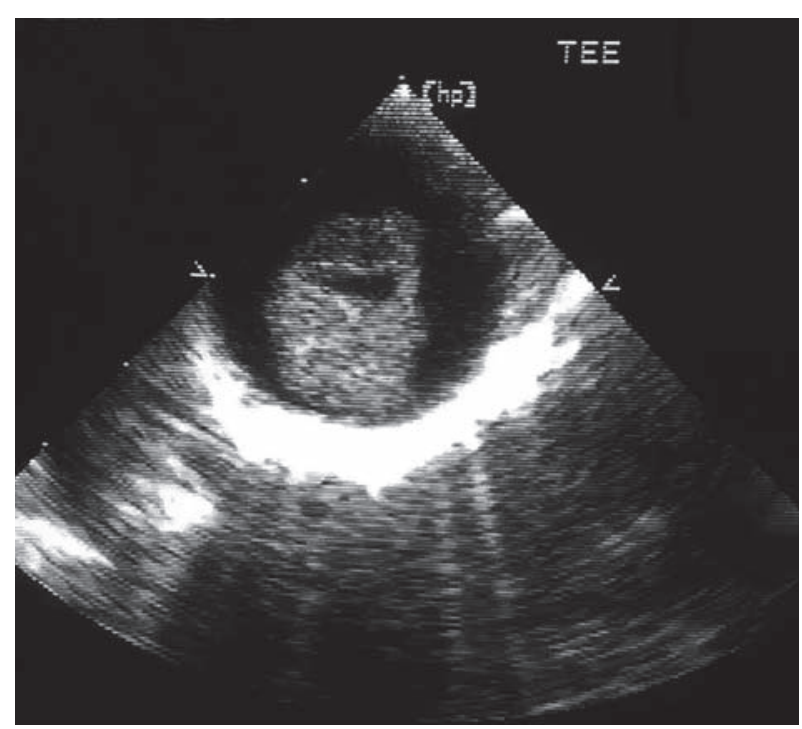

Figura 2. Ecocardiografía transesofágica al ingreso. Flecha roja: masa móvil de gran tamaño en aorta descendente.

cuanto a la medicación, únicamente se encontraba en tratamiento con anticonceptivos hormonales orales. Al examen físico, se constató ausencia de pulso en MSI con disminución del relleno capilar y de la temperatura por lo que se decidió evaluación mediante eco-Doppler arterial de miembro superior izquierdo que evidencia oclusión de las arterias axilar, humeral, radial y cubital. Ante dichos hallazgos, se realizó un análisis de enfermedades trombofílicas, que resultó negativo, y un ETE (Figura 2) y angiotomografía (Figura 3), los cuales informaron una aorta descendente no dilatada sin evidencia de disección, con extensa masa móvil de $21 \times 10 \times 12$ mm y anclaje distal más oclusión de arteria subclavia izquierda desde ostium hasta tercio medio de la arteria humeral izquierda. Por las imágenes de los estudios complementarios, se planteó el diagnóstico diferencial entre trombo y angiosarcoma, por lo que se la evaluó mediante una tomografía con emisión de positrones que no evidenció aumento de la captación en la aorta torácica (Figura 4). Con la sospecha de diagnóstica de trombo se suspendió la cirugía ginecológica, se inhibió farmacológicamente el ciclo menstrual y se inició anticoagulación hasta definir conducta. Durante la internación evolucionó con dolor en miembro inferior derecho con disminución de los pulsos en el mismo, por lo que se realizó eco-Doppler arterial de miembro inferior derecho que evidenció oclusión de la arteria poplítea derecha.

Encontrándose en tratamiento anticoagulante, se realizó nuevo ETE a las 3 semanas que no evidenció trombo. Posteriormente se le realizó una histerectomía laparoscópica, evolucionando favorablemente en el posoperatorio. Fue externada a los 10 días con anticoagulación oral.

\section{DISCUSIÓN}

La fisiopatología de los trombos aórticos no está claramente definida y su diagnóstico es usualmente a partir de una embolización periférica. Son más frecuentes en pacientes de edad avanzada o con múltiples factores de riesgo cardiovasculares y arteriosclerosis aórtica severa ${ }^{1,6,7}$. En los casos de masas móviles en una aorta previamente sana pueden ser resultado de una enfermedad de hipercoagulabilidad primaria causada por déficit

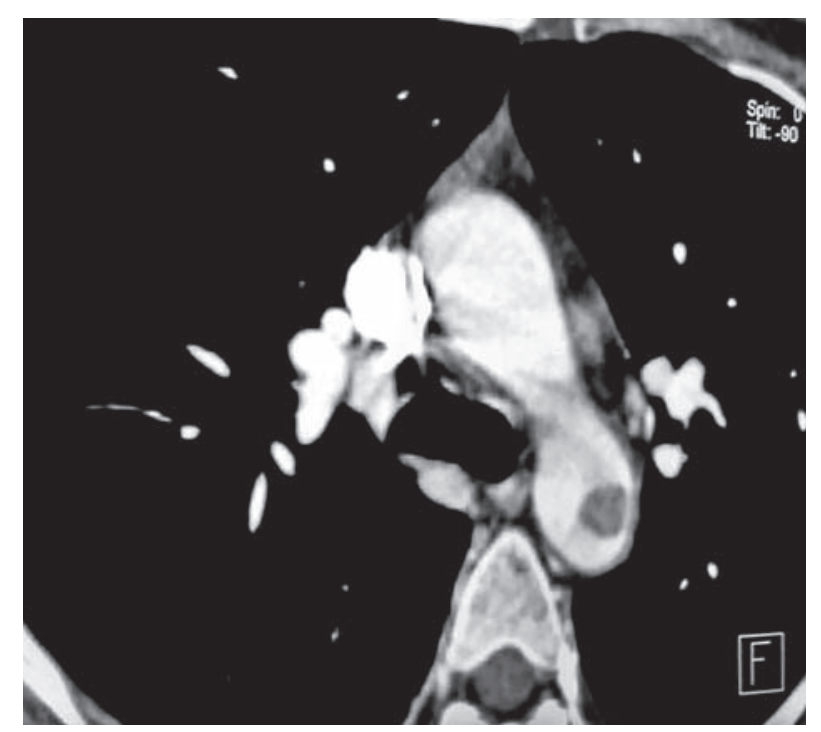

Figura 3. Angiotomografía. Flecha roja: masa en aorta descendente.
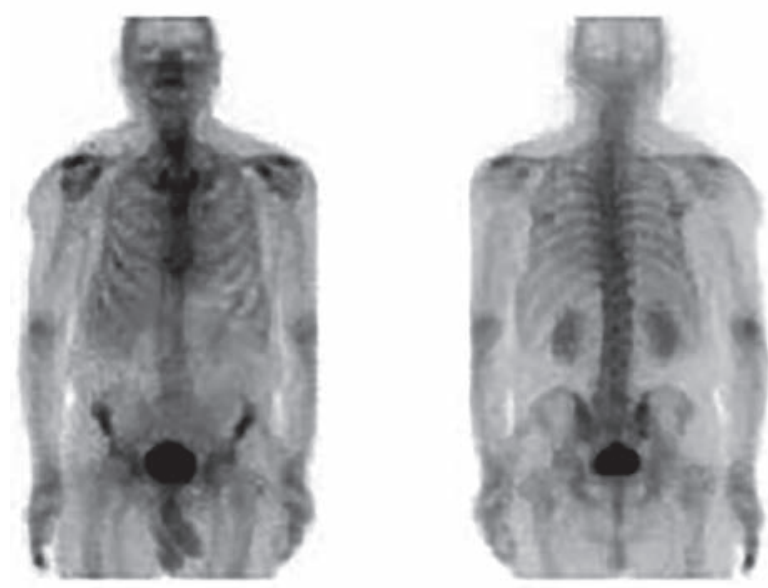

Figura 4. Tomografía con emisión de positrones sin evidencia de aumento de captación en la zona de la aorta

de uno o más componentes del sistema de anticoagulación, o un desorden agudo secundario a lupus, terapia con estrógenos, reacciones inmunológicas hacia la heparina, entre otros. El antecedente familiar de alteraciones trombofilicas sugiere un desorden del sistema de coagulación $n^{5,6}$

Es necesario realizar el diagnóstico diferencial con otras masas aórticas, como es el caso de los tumores. El diagnóstico definitivo requiere del estudio histológico e inmunohistoquímico, sin embargo la resonancia magnética es la prueba por imágenes más útil para este diagnóstico diferencial, y la tomografía con emisión de positrones ${ }^{8}$. También es importante contemplar la posible disección aórtica por las implicaciones terapéuticas.

Para el diagnóstico de esta patología es fundamental la realización de un ETE dado tiene una elevada exactitud diagnóstica y permite valorar el tamaño, la morfología y el punto de anclaje del trombo, así como las características de la pared aórtica ${ }^{1,6}$. También es importante la angiotomografía para la ubicación exacta en casos quirúrgicos ${ }^{4}$. 
La piedra angular del tratamiento de esta patología es la anticoagulación. Un período de aproximadamente dos semanas es recomendado para realizar un nuevo ETE o angiotomografía para evaluar el éxito terapéutico de la anticoagulación $n^{5,6}$.

Si el paciente tiene un riesgo quirúrgico aceptable con trombo de alta movilidad y falta de disminución del tamaño a pesar del tratamiento anticoagulante o repite nuevos episodios embólicos, se sugiere la conducta quirúrgica temprana a menos que el trombo tenga menos de $1 \mathrm{~cm}$ de tamaño ${ }^{5}$. Se recomienda anticoagulación oral por un año más aspirina después de la trombectomía. En caso de que el paciente presente historia familiar de trombosis, se recomienda anticoagulación oral de por vida ${ }^{5}$.

\section{BIBLIOGRAFÍA}

1. Dee W, Geibel A, Kasper W, Konstantinides S, Just H. Mobile thrombi in atherosclerotic lesions of the thoracic aorta: the diagnostic impact of transesophageal echocardiography. Am Heart J 1993;126(3):707-10.

2. Reber PU, Patel AG, Stauffer E, Müller MF, Do DD, Kniemeyer HW. Mural aortic thrombi: An important cause of peripheral embolization. I Vasc Surg 1999;30(6):1084-9.

3. Laraudogoitia E, Evangelista A, García del Castillo H, Lekuona I, Palomar S, González-Alujas T, et al. Trombo en aorta torácica como causa de embolismo periférico diagnosticado por ecocardiografía transesofágica. Rev Esp Cardiol 1997;50(1):62-4.

4. Hahn TL, Dalsing MC, Sawchuk AP, Cikrit DF, Lalka SG. Primary aortic mural thrombus: presentation and treatment. Ann Vasc Surg 1999;13(1):52-9.

\section{CONCLUSIÓN}

En pacientes con embolia arterial, además de descartar focos de embolia habituales es conveniente investigar la aorta torácica (ETE y/o angiotomografía), incluso en sujetos sin factores de riesgo cardiovasculares clásicos. Ante la duda diagnóstica entre angiosarcoma y trombo se recomienda continuar evaluación mediante tomografía con emisión de positrones o una resonancia magnética para descartar proceso maligno que obligue a un abordaje quirúrgico inmediato. La anticoagulación oral sigue siendo la estrategia recomendada ante la presencia de trombos.

5. Choukroun EM, Labrousse LM, Madonna FP, Deville C. Mobile thrombus of the thoracic aorta: diagnosis and treatment in 9 cases. Ann Vasc Surg 2002;16(6):714 22.

6. Laperche T, Laurian C, Roudaut R, Steg PG. Mobile thromboses of the aortic arch without aortic debris. A transesophageal echocardiographic finding associated with unexplained arterial embolism. The Filiale Echocardiographie de la Société Française de Cardiologie. Circulation 1997;96(1):288-94.

7. Patra P, Pillet JC, Chaillou P, Duveau D. Traitement chirurgical des lesions emboligenes de la crosse de l'aorte. In: Ed. Pharmapost, Paris, 1997 pp 11-31.

8. Thalheimer A, Fein M, Geissinger E, Franke S. Intimal angiosarcoma of the aorta: report of a case and review of the literature. J Vasc Surg 2004;40(3):548-53. 